\title{
Variación morfológica de plántulas e influencia del pH del agua de riego en doce poblaciones de Pinus greggii Engelm.
}

\author{
Javier López Upton ${ }^{1}$ \\ Adrián J. Mendoza Herrera \\ Jesús Jasso Mata ${ }^{1}$ \\ J. Jesús Vargas Hernández ${ }^{1}$ \\ Armando Gómez Guerrero ${ }^{1}$
}

\section{RESUMEN}

Se estudiaron plántulas de 16 meses de edad de Pinus greggii bajo condiciones de invernadero para determinar si existía variación geográfica en relación con la acidez del suelo. El estudio incluyó cinco familias de polinización libre de cada una de 12 poblaciones probadas. Se encontraron diferencias significativas tanto a nivel de poblaciones como a nivel de familias en los días para emergencia, color del follaje y altura total. Se determinó una clara separación de las poblaciones en dos grandes grupos, uno de la parte norte y otro de la sur de la distribución natural de la especie. Estos resultados concuerdan con la información recientemente publicada de la descripción de una nueva variedad de $P$. greggii. Además se observó un control genético de alto a moderado en altura total y en el color del follaje, lo que posibilita una selección temprana a nivel familial e individual de las mejores poblaciones.

PALABRAS CLAVE:

Pinus greggii, var. greggii, var. australis, procedencias, ph del suelo, heredabilidad.

\begin{abstract}
A greenhouse study on sixteenmonth-old seedlings of Pinus greggii was carried out to determine whether geographic variation exists in relation to soil acidity. The study included five openpollinated families from each of twelve populations. Significant differences were found both among and within populations for seedling emergence, leaf color and total height. A pattern was detected in relation to geographic location. Northern populations as a group showed different treatment effects than the southern populations. This result agrees with information recently published in the description of a new variety of the species. Seedling height and leaf color were found to be under genetic control which may be useful for early selection of families within superior provenances.
\end{abstract}

KEY WORDS:

Pinus greggii, var. greggii, var. australis, provenances, soil $\mathrm{pH}$, hereditability.

1 Especialidad Forestal. Instituto de Recursos Naturales. Colegio de Postgraduados. Montecillo, Edo. de México. 56230 México. c.e.: uptonj@colpos.colpos.mx.

Manuscrito recibido el 7 de marzo del 2000. Aceptado el 8 de mayo del 2000. 


\section{INTRODUCCION}

Una especie de conífera de reciente y amplio uso ha sido Pinus greggii Engelm. debido a su rápido crecimiento y buena adaptación a ambientes diferentes. Las poblaciones naturales de $P$. greggii se encuentran localizadas en pequeños manchones a lo largo de la Sierra Madre Oriental, aisladas por barreras geográficas que limitan el intercambio genético entre ellas. Además se ha detectado cierto grado de variación entre y dentro de procedencias 0 poblaciones (Malagón 1990; López et al. 1993), incluso variaciones en el crecimiento con relación al $\mathrm{pH}$ del suelo (Donahue 1990).

Ruiz (1989) encontró variación significativa en características juveniles entre y dentro de dos poblaciones en tres tipos de sustratos y $\mathrm{pH}$. Mientras que García (1990) recomienda para un buen desarrollo de $P$. greggii en vivero un $\mathrm{pH}$ cercano a 5.8. Por su parte, Donahue (1990), analizando el crecimiento de seis poblaciones en dos sustratos, uno ácido y otro alcalino encontró diferencias, al parecer, en relación al pH del suelo en los lugares de origen. Dvorak y Donahue (1993) señalan que las poblaciones del noreste y centro-este del país (norte y sur de su distribución natural) pueden representar dos variedades diferentes, debido a que en su medio nativo parecen estar adaptadas a condiciones ambientales diferentes. Recientemente, Donahue y López (1999) después de realizar análisis morfológicos de hojas, conos y semillas de 12 poblaciones y observar el crecimiento en ensayos en un sólo ambiente separaron la especie en dos variedades, $P$. greggii Engelm. var. greggii, que corresponden las poblaciones del noreste de México, y $P$. greggii var. australis Donahue \& Lopez a las poblaciones del sur de su distribución natural.

Por otro lado, dentro de un mismo taxón se pueden encontrar grandes diferencias de comportamiento respecto al lugar de procedencia. Este fenómeno inicia desde el momento de la germinación, lo que implica que se pueda realizar una selección temprana de los mejores individuos de los mejores orígenes, si esta característica está relacionada con alguna otra a edad madura o es una característica que sea útil en la sobrevivencia de las plantas a edad temprana. Por ejemplo, Lutz y Chandler (1949) indican que los efectos de los valores de $\mathrm{pH}$ del suelo son más evidentes en las plántulas que en árboles maduros.

\section{OBJETIVOS}

Debido a lo anterior, se inició la presente investigación para estimar la variación genética a nivel plántula entre y dentro de doce poblaciones de $P$. greggii que abarcan su área de distribución natural. Otro objetivo es cuantificar el grado de control genético para la altura y color del follaje en plántulas estimando la heredabilidad individual y de promedios de familias. Además, observar el comportamiento debido a cambios de $\mathrm{pH}$ del agua de riego y suelo durante su crecimiento en vivero.

\section{METODOLOGÍA}

El experimento se realizó en un invernadero del Colegio de Postgraduados en Montecillo, Edo. de México; localizado en las coordenadas $19^{\circ} 28^{\prime} 05^{\prime \prime}$ L.N. y 98 54'09" L.W., a una altitud de 2220 msnm. Se utilizó semilla de doce poblaciones (Tabla 1), cada una con cinco árboles, que se mantuvieron separados como familias de polinización libre. En cada sitio de colecta, la selección de los árboles se efectuó con base en el método comparativo, procurando que fuesen vigorosos, sanos y fenotípicamente aceptables (igual o mejor al promedio de la población). La separación entre árboles fue mayor a $100 \mathrm{~m}$. 
Tabla 1. Características de los sitios donde proceden las poblaciones en estudio de Pinus greggii.

\begin{tabular}{|l|l|c|c|c|c|c|c||}
\hline \multicolumn{2}{||c|}{ POBLACIÓN ${ }^{(1)}$} & L.N. & L.W. & $\begin{array}{c}\text { ALTITUD } \\
\text { msnm }\end{array}$ & $\begin{array}{c}\text { PP } \\
(\mathrm{mm})^{(2)}\end{array}$ & $\begin{array}{c}\text { T } \\
\left({ }^{\circ} \mathrm{C}\right)^{(2)}\end{array}$ & $\begin{array}{c}\text { pH } \\
\text { SUELO }^{(3)}\end{array}$ \\
\hline 1. & Puerto Conejos, N.L.-Coah. & $25^{\circ} 28^{\prime}$ & $100^{\circ} 35^{\prime}$ & 2400 & 650 & 13 & 6.0 \\
\hline 2. & Santa Anita, Coah & $25^{\circ} 27^{\prime}$ & $100^{\circ} 35^{\prime}$ & 2500 & 650 & 13 & 6.8 \\
\hline 3. & Puerto San Juan, Coah & $25^{\circ} 25^{\prime}$ & $100^{\circ} 33^{\prime}$ & 2650 & 600 & 13 & 6.9 \\
\hline 4. & Los Lirios, Coah. & $25^{\circ} 24^{\prime}$ & $100^{\circ} 34^{\prime}$ & 2350 & 600 & 12 & 7.4 \\
\hline 5. & Jamé, Coah & $25^{\circ} 01^{\prime}$ & $100^{\circ} 36^{\prime}$ & 2370 & 600 & 13 & 7.2 \\
\hline 6. & Las Placetas, N.L. & $24^{\circ} 54^{\prime}$ & $100^{\circ} 12^{\prime}$ & 2250 & 750 & 16 & 7.2 \\
\hline 7. & El Madroño, Qro. & $21^{\circ} 16^{\prime}$ & $99^{\circ} 09^{\prime}$ & 1750 & 1200 & 17 & 4.5 \\
\hline 8. & El Piñón, Hgo. & $20^{\circ} 57^{\prime}$ & $99^{\circ} 13^{\prime}$ & 1830 & 850 & 18 & 5.6 \\
\hline 9. & Molango, Hgo. & $20^{\circ} 48^{\prime}$ & $98^{\circ} 44^{\prime}$ & 1400 & 1500 & 16 & 4.4 \\
\hline 10. & Xochicoatlán, Hgo. & $20^{\circ} 47^{\prime}$ & $98^{\circ} 42^{\prime}$ & 1850 & 1750 & 17 & 5.4 \\
\hline 11. & Minas San Fco., Hgo & $20^{\circ} 45^{\prime}$ & $98^{\circ} 22^{\prime}$ & 1845 & 650 & 18 & 5.2 \\
\hline 12 & Patoltecoya, Pue. & $20^{\circ} 13^{\prime}$ & $98^{\circ} 02^{\prime}$ & 1415 & 2000 & 20 & 5.6 \\
\hline
\end{tabular}

1 Poblaciones 1 a 6 corresponden a P. greggii Engelm var. greggii; poblaciones 7 a 12 corresponden a $P$. greggii var. australis Donahue \& Lopez.

2 Precipitación total anual y temperatura media anual. Fuente : INEGI (1980).

3 Muestras en campo determinadas en el laboratorio de Física de Suelos, IRENAT-CP.

Se utilizaron envases de polietileno de $3.0 \mathrm{~cm}$ de diámetro por $17.5 \mathrm{~cm}$ de alto, con capacidad de 100 cc y de forma cónica (contenedor). El sustrato que se utilizó fue una mezcla $50 \%$ de tierra de monte y $50 \%$ hojarasca de encino debidamente picada y cernida, con un $\mathrm{pH}$ de 5.7 y una conductividad eléctrica (C.E.) de $0.65 \mathrm{mmhos} / \mathrm{cm}$. Las semillas, previamente remojadas por 24 horas, se sembraron el 24 de noviembre de 1992 a una profundidad de $1.5 \mathrm{~cm}$. Hasta los ocho meses de edad se utilizó para regar agua con un pH de 7.5 (la que normalmente se obtiene en el área de Montecillo, Edo. de Méx.), lo que originó una disminución de la acidez del suelo $(\mathrm{pH}=6.1)$ y un aumento de la C.E. a 5.2. Posteriormente, utilizando una solución de ácido sulfúrico se disminuyó el pH del agua a 5.2, riego que se mantuvo hasta los dieciséis meses de edad de la planta, lo que aumentó la acidez del sustrato a 5.9 de $\mathrm{pH}$ y una disminución de la C.E. a $1.78 \mathrm{mmhos} / \mathrm{cm}$. Se utilizó ácido fosfórico porque es un ácido de uso seguro, no reacciona violentamente con el agua, ni produce vapores tóxicos y proporciona fósforo (Landis et al. 1989).

Se utilizó un diseño de cinco bloques al azar y un arreglo de parcelas divididas. Dentro de cada bloque se colocaron las doce poblaciones distribuidas al azar y dentro de cada una de ellas las cinco familias con veinte plantas por parcela experimental $(5 \times 12 \times 5 \times 20=6000$ plántulas). Se consideraron las variables: Días para la emergencia a 50\%, altura total de la planta, color del follaje $y$ sobrevivencia.

La variable días para la emergencia a $50 \%$ de plántulas se obtuvo 
registrándose los días que tardó cada plántula en emerger desde el momento de la siembra, analizándose el valor cuando se alcanzó el $50 \%$ de la emergencia total de plántulas. La altura total se evaluó a 8 y 16 meses con una regla graduada, midiéndose desde la base a la yema apical de la planta. El color del follaje se evaluó por medio de una tabla de colores de Séguy (1936), desde verde obscuro (valor 1,387 en Séguy) hasta verde amarillento (valor 9,257 en Séguy). Cuando un tono se identificaba como intermedio entre dos valores se agregó al del inferior la cantidad de 0.5 , lo que dio 17 tonos diferentes. La sobrevivencia se analizó a 16 meses, contabilizándose el número de plántulas sobrevivientes en función a las emergidas.

Primeramente se realizó un análisis de normalidad usando el procedimiento UNIVARIATE con la opción NORMAL (SAS 1988) encontrándose que, a excepción de la sobrevivencia, las variables pertenecían a la muestra de una población normal. Para la sobrevivencia, dada su baja normalidad se realizó una transformación arcoseno $(\%)^{1 / 2}, \quad$ y se analizó a nivel de poblaciones con el procedimiento ANOVA del SAS. La variable días para la emergencia se transformó con logaritmo base 10 para normalizar la variable. Sobrevivencia y emergencia se analizaron como valores medios de parcela usando el modelo (1), pero incluyendo el efecto de $B F(P)$ en el error total.

El color y altura de plántulas se analizaron con valores individuales. Debido a la pérdida involuntaria de algunos datos de color y altura, se utilizó el procedimiento GLM del SAS (SAS 1988), y un modelo jerárquico con todos los efectos aleatorios (Modelo 1). La esperanza de los cuadrados medios del modelo 1 se indican en la Tabla 2. Sin embargo, para realizar la prueba de medias de poblaciones se realizó a través de una prueba de Tukey, considerándose al efecto de poblaciones como fijo.

$$
\begin{aligned}
& Y_{i j k n}=\mu+B_{i}+P_{j}+B P_{i j}+F(P)_{j k}+B F(P)_{j i k}+E_{i j k n} \\
& \text { (Modelo 1) } \\
& \text { Donde: } \\
& Y_{\mathrm{ijkn}}=\text { observación de la n-ésima } \\
& \mu \quad=\text { media poblacional } \\
& \mathrm{B}_{\mathrm{i}}=\text { efecto aleatorio de bloque } \\
& \sim N I D\left(0, \sigma^{2}\right) \\
& \mathrm{P}_{\mathrm{j}} \quad=\text { efecto aleatorio de procedencia } \\
& \sim N I D\left(0, \sigma_{p}^{2}\right) \\
& \mathrm{BP}_{\mathrm{ij}}=\text { efecto aleatorio de la } \\
& \text { interacción procedencia por } \\
& \text { bloque } \sim N I D\left(0, \sigma^{2} \text { bp }\right) \\
& \mathrm{F}(\mathrm{P})_{\mathrm{jk}}=\text { efecto aleatorio de familia } \\
& \text { anidada en procedencia } \sim N I D \\
& \left(0, \sigma_{f(p)}^{2}\right) \\
& \mathrm{BF}(\mathrm{P})_{\mathrm{ijk}}=\begin{array}{l}
\text { efecto aleatorio de la } \\
\text { interacción de familia por }
\end{array} \\
& \text { bloque } \sim N I D\left(0, \sigma^{2}{ }_{b f(p)}\right) \\
& \mathrm{E}_{\mathrm{ijkn}}=\text { término del error } \sim N I D\left(0, \sigma^{2}{ }_{\mathrm{e}}\right)
\end{aligned}
$$

Las familias son de polinización libre, por lo que se consideran de medios hermanos, de ahí que el componente de varianza para familias $\left(\sigma_{\mathrm{f}}^{2}\right)$ puede ser interpretado como un estimado de un cuarto de la varianza genética aditiva $\left(\sigma^{2}{ }_{\mathrm{A}}\right)$ (Falconer y Mackay 1996). Así, se calculó la heredabilidad a nivel de individuos $\left(h^{2}\right)$ y a nivel de familias $\left(\mathrm{h}_{f}^{2}\right)$ de las variables color y altura, a través de las fórmulas siguientes (Zobel y Talbert 1984):

$$
\begin{aligned}
& \begin{array}{l}
h^{2}=\left[4^{*} \sigma_{f(p)}^{2}\right] /\left[\sigma_{e}^{2}+\sigma_{b f(p)}^{2}+\sigma_{f(p)}^{2}\right] \\
(\text { Modelo 2) }
\end{array} \\
& \begin{array}{r}
h_{f}^{2}=\left[\sigma_{f(p)}^{2}\right] /\left[\sigma^{2}{ }_{e} / n b+\sigma_{b f(p)}^{2} / b+\sigma_{f(p)}^{2}\right] \\
(\text { Modelo 3) }
\end{array} \\
& \begin{aligned}
n= & \text { fue el valor de la media armónica } \\
& \text { del número de plantas por } \\
& \text { parcela, y de bloques. }
\end{aligned} \\
& b=\text { el número de bloques. }
\end{aligned}
$$

Donde: 
Para obtener los valores de las varianzas de familias $\left(\sigma_{\mathrm{f}(\mathrm{p})}^{2}\right)$ y de la interacción de familias por bloque $\left(\sigma^{2}\right.$ bf(p) $)$ se realizaron varias conversiones matemáticas de los valores obtenidos en el análisis de varianza basado en la suma de cuadrados tipo III y en los cuadrados medios esperados indicados en la Tabla 2.

\section{RESULTADOS Y DISCUSIÓN}

\section{Días para la emergencia.}

Se encontró una variación altamente significativa de la emergencia de plántulas entre poblaciones y entre las familias dentro de poblaciones. En los valores medios de las poblaciones (Tabla 3, Fig. 1), se observó una separación de acuerdo con las variedades taxonómicas propuestas por Donahue y López (1999), ya que las plántulas de la var. greggii germinan y emergen más rápido que las de la var. australis. Se vislumbra cierto control genético, que puede ser una adaptación a la menor duración de la estación de crecimiento (Squillace 1966) en Coahuila y Nuevo León, y que estas poblaciones tienen semillas más grandes y y pesadas (Malagón 1990; López et al. 1993), las cuales deben dar plántulas más vigorosas para germinar y emerger con las primeras lluvias e iniciar el desarrollo rápidamente en estas zonas de menor periodo de crecimiento.

Cabe señalar que los valores promedio del número de días para obtener la emergencia del $50 \%$ de plántulas resultó bastante alto, lo cual pudo deberse a las temperaturas relativamente bajas del invierno durante el cual se sembró $\left(15^{\circ} \mathrm{C}\right.$ de temperatura media durante este periodo en el invernadero), además a que la siembra se realizó un poco profunda y sin estratificación a la semilla.

En algunas especies de coníferas se ha encontrado que las poblaciones de los sitios más secos y con estaciones de crecimiento más cortas producen semillas de mayor tamaño y con mayor velocidad de germinación. Ejemplo de ello es lo encontrado por Ruiz y Velasco (1994) en $P$. engelmanii Carr. y por Sorensen y Miles (1978) en Pseudotsuga menziesii (Mirb.) Franco, los que concluyeron que las semillas más grandes son aptas para

Tabla 2. Cuadrados medios esperados para el análisis de color del follaje y altura de las plántulas en el ensayo de poblaciones/progenies de polinización libre de Pinus greggii.

\begin{tabular}{|c|c|c|}
\hline FUENTE DE VARIACIÓN & G. L. & CUADRADOS MEDIOS ESPERADOS \\
\hline Bloque & $b-1$ & $\sigma_{e}^{2}+n \sigma_{b f(p)}^{2}+n f \sigma_{b p}^{2}+n f p \sigma_{b}^{2}$ \\
\hline Procedencia & $p-1$ & $\sigma_{e}^{2}+n \sigma_{b f(p)}^{2}+n f \sigma_{b p}^{2}+n b \sigma_{f(p)}^{2}+n f b \sigma_{p}^{2}$ \\
\hline Bloque $x$ Procedencia & $(b-1)(p-1)$ & $\sigma_{e}^{2}+n \sigma_{b f(p)}^{2}+n f \sigma_{b p}^{2}$ \\
\hline Familia (Procedencia) & $(f-1) p$ & $\sigma_{e}^{2}+n \sigma_{b f(p)}^{2}+n b \sigma_{f(p)}^{2}$ \\
\hline Bloque x Familia (Procedencia) & $(b-1)(f-1) p$ & $\sigma_{e}^{2}+n \sigma_{b f(p)}^{2}$ \\
\hline Error & $(n-1) b p f$ & $\sigma_{e}^{2}$ \\
\hline Total & bpfn-1 & \\
\hline $\begin{array}{l}\text { Jonde: } \\
\text { G.L. = grados de libertad } \\
\mathrm{b}=\text { número de bloques }\end{array}$ & & $\begin{array}{l}=\text { número de familias } \\
=\text { número de plantas por parcela. }\end{array}$ \\
\hline
\end{tabular}

$\mathrm{p}$ = número de poblaciones

Se consideró a todos los efectos como aleatorios. 
Tabla 3. Valores medios de seis variables de plántulas de 12 poblaciones de Pinus greggii.

\begin{tabular}{|c|c|c|c|c|c|c|}
\hline \multirow{2}{*}{ POBLACIÓN ${ }^{1}$} & \multirow{2}{*}{$\begin{array}{l}\text { EMER } \\
\text { GENCIA } \\
(2)\end{array}$} & \multicolumn{2}{|c|}{$\begin{array}{l}\text { COLOR DEL } \\
\text { FOLLAJE }^{(3)}\end{array}$} & \multicolumn{2}{|c|}{$\begin{array}{l}\text { ALTURA TOTAL } \\
(\mathrm{cm})\end{array}$} & \multirow{2}{*}{$\begin{array}{l}\text { SOBREVI- } \\
\text { VENCIA }{ }^{(4)}\end{array}$} \\
\hline & & 8 meses & $\begin{array}{c}16 \\
\text { meses }\end{array}$ & 8 meses & 16 meses & \\
\hline 1. Puerto Conejos, N.L.-Coah. & $33 \mathrm{f}$ & 3.7 ef & $3.1 \mathrm{~cd}$ & $8.4 \mathrm{f}$ & 13.3 ef & $83.4 \mathrm{a}$ \\
\hline 2. Santa Anita, Coah. & $33 \mathrm{f}$ & $3.7 \mathrm{fg}$ & $3.2 \mathrm{c}$ & 8.7 ef & $14.0 \mathrm{de}$ & $87.4 \mathrm{a}$ \\
\hline 3. Pto. San Juan, Coah. & $33 \mathrm{f}$ & $3.4 \mathrm{~h}$ & $2.9 \mathrm{~d}$ & $8.5 \mathrm{f}$ & $14.5 d$ & $86.2 \mathrm{a}$ \\
\hline 4. Los Lirios, Coah. & $30 \mathrm{~g}$ & $3.5 \mathrm{gh}$ & $3.2 \mathrm{c}$ & $8.5 \mathrm{f}$ & $13.1 \mathrm{f}$ & $87.8 \mathrm{a}$ \\
\hline 5. Jamé, Coah. & $32 \mathrm{fg}$ & $3.5 \mathrm{gh}$ & $3.1 \mathrm{~cd}$ & 8.8 ef & $14.7 \mathrm{~d}$ & $88.8 \mathrm{a}$ \\
\hline 6. Las Placetas, N.L. & $31 \mathrm{fg}$ & $3.3 \mathrm{~h}$ & $3.2 \mathrm{c}$ & $9.6 \mathrm{~d}$ & $14.4 \mathrm{~d}$ & $89.0 \mathrm{a}$ \\
\hline 7. El Madroño, Qro. & $52 \mathrm{~b}$ & $3.9 \mathrm{~cd}$ & $4.0 \mathrm{a}$ & $12.7 \mathrm{a}$ & $23.0 \mathrm{a}$ & $93.2 \mathrm{a}$ \\
\hline 8. El Piñón, Hgo. & $42 \mathrm{~d}$ & $3.8 \mathrm{de}$ & $4.0 \mathrm{a}$ & $12.2 \mathrm{ab}$ & $20.7 \mathrm{~b}$ & $95.8 \mathrm{a}$ \\
\hline 9. Molango, Hgo. & $56 \mathrm{a}$ & $4.4 \mathrm{~b}$ & $3.9 \mathrm{ab}$ & $11.2 \mathrm{c}$ & $21.2 \mathrm{~b}$ & $83.2 \mathrm{a}$ \\
\hline 10. Xochicoatlán, Hgo. & $47 \mathrm{c}$ & $4.1 \mathrm{c}$ & $3.7 \mathrm{~b}$ & $11.1 \mathrm{c}$ & $21.5 \mathrm{~b}$ & $83.4 \mathrm{a}$ \\
\hline 11. Minas San Fco., Hgo & $35 \mathrm{e}$ & $4.0 \mathrm{~cd}$ & $4.1 \mathrm{a}$ & $9.3 \mathrm{de}$ & $16.7 \mathrm{c}$ & $41.6 \mathrm{c}$ \\
\hline 12. Patoltecoya, Pue. & $46 \mathrm{c}$ & $5.1 \mathrm{a}$ & $4.1 \mathrm{a}$ & $11.9 \mathrm{~b}$ & $20.9 \mathrm{~b}$ & $64.8 \mathrm{~b}$ \\
\hline
\end{tabular}

\footnotetext{
1 Las poblaciones 1 a 6 corresponden a $P$. greggii Engelm. var. greggii y de la 7 a 12 corresponden a $P$. greggii var. australis Donahue \& Lopez.

2 Días para obtener el $50 \%$ de emergencia de plántulas.

3 Valores mayores significan tonos más amarillentos.

$4 \quad$ Evaluación a 16 meses de edad en porcentaje.

Letras iguales no son significativamente diferentes con $p=0.05$.
}

Días a partir de la siembra

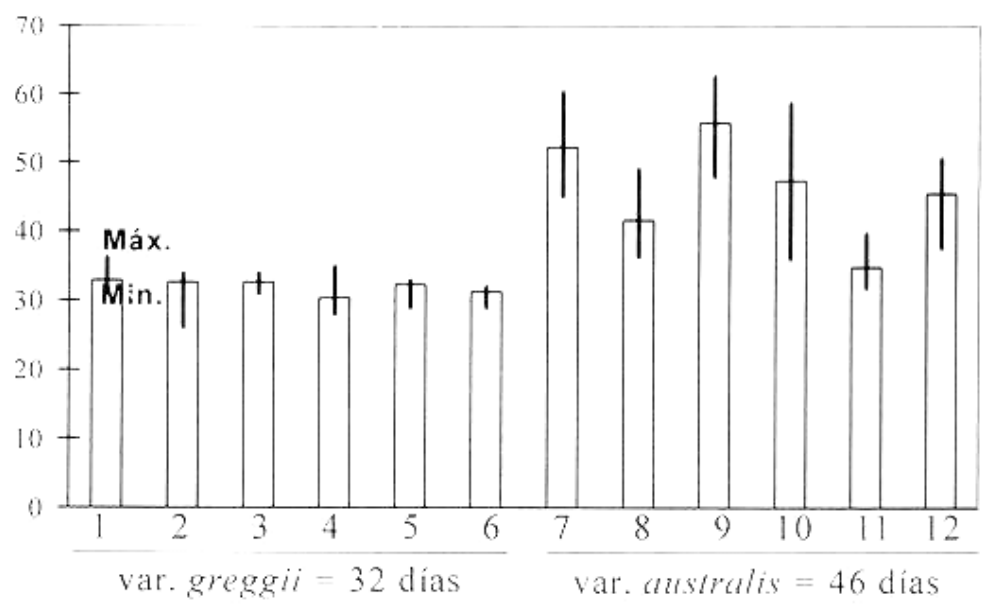

Poblaciones

Figura 1. Valores promedio por procedencia (barra) y promedios de las familias extremas (Mín. y Máx.) para la variable $50 \%$ de emergencia de plántulas de Pinus greggii. El análisis de varianza indicó diferencias significativas con $p<0.01$ entre poblaciones y familias. Poblaciones: 1) Puerto Los Conejos; 2) Santa Anita; 3) Puerto San Juan; 4) Los Lirios; 5) Jamé; 6) El Madroño; 7) El Piñón; 9) Molango; 10) Xochicoatlán; 11) Minas San Francisco y 12) Patoltecoya. 
regenerar sitios donde la estación de crecimiento es corta. En concordancia con la hipótesis anterior, se observa que dentro de las poblaciones del sur (var. australis), la muestra de semilla colectada en Minas San Francisco, Hgo. resultó ser la más rápida en emerger y ésta es la población de menor precipitación dentro de esta variedad.

Los valores mínimos y máximos de las familias dentro de cada población reflejan las diferencias que existen entre las familias dentro de poblaciones (Fig. 1). Por ejemplo, en la var. australis, en Xochicoatlán, Hgo. se presentaron una familia con un promedio de 36 y otra con 58 días para el $50 \%$ de emergencia, valores mínimos y máximos por familia respectivamente, lo que significa 22 días de diferencia, lo que demuestra la variación que existe entre familias dentro de las poblaciones. También se observa que a nivel familia el valor mínimo lo tuvo Minas San Francisco, Hgo. con 34 y el máximo Molango, Hgo. con 63 días, rango que representa amplia diferencia (29 días) de las familias dentro de la var. australis. En el grupo norte (var. greggii) las diferencias entre familias resultaron menos drásticas, debido a que su valor mínimo corresponde a Jamé, Coah. con 29 días y el máximo a Puerto Los Conejos, Coah.N.L. con 36 días, cuya diferencia es cerca de 7 días en todo el grupo.

\section{Sobrevivencia}

La sobrevivencia se evaluó hasta los 16 meses después de la siembra encontrándose diferencias altamente significativas entre poblaciones, lo cual indica una variabilidad de la especie para sobrevivir en las primeras etapas en vivero de acuerdo a la procedencia y después de las modificaciones en los valores de $\mathrm{pH}$ del suelo (Tabla 3).

Las poblaciones con menor sobrevivencia resultaron Patoltecoya, Pue. (65\%) y Minas San Francisco, Hgo. (42\%). Ambas poblaciones habitan en suelos ácidos lo que puede indicar su baja adaptabilidad a suelos de $\mathrm{pH}$ neutro. Probablemente también se presentan algunos problemas de endogamia, debido a lo pequeño de sus poblaciones, factor que se puede manifestar en el vigor de las plántulas. Estudios realizados en especies de coníferas y otras especies con mecanismos de polinización cruzada muestran que en poblaciones aisladas con reducido número de individuos, es considerable la frecuencia de autopolinización y con ello la endogamia (Sorensen y Miles 1978; Matheson et al. 1989). Esta condición trae como consecuencia, entre otras cosas, una reducción en el vigor y crecimiento de las plántulas (Sorensen y Miles 1978).

\section{Color del follaje}

En el análisis de varianza para el color a 8 y 16 meses de edad, se encontraron diferencias altamente significativas entre poblaciones y entre familias dentro de las poblaciones. En los sustratos utilizados para producir plantas forestales en vivero se recomienda tener $\mathrm{pH}$ ácidos de lo contrario se corre el riesgo de que las plántulas sean atacadas por damping-off, otros organismos dañinos, o bien que las plántulas manifiesten una clorosis específicamente por falta de disponibilidad del fierro (Pritchett 1986). El síntoma de clorosis se consideró para estudiar el comportamiento de las poblaciones de $P$. greggii en $\mathrm{pH}$ ligeramente básico en el sustrato del vivero. A los 8 meses de edad, con pH mayor, se verificó la separación de la var. greggii (poblaciones del norte) y de la var. australis (del sur de su distribución natural), las poblaciones del segundo grupo manifestaron una clorosis 0 amarillamiento mayor que las del primero, debido posiblemente a que en su hábitat natural, $P$. greggii se desarrolla en $\mathrm{pH}$ ácidos (Tabla 1).

En la figura 2 también se observan diferencias en el color del follaje a 8 meses de edad de las familias dentro de las 
poblaciones. En el grupo var. australis, la población con la mayor diferencia entre familias resultó Patoltecoya, Pue. con 1.58 unidades entre el valor mínimo y máximo de sus familias. Desde una perspectiva general dentro del grupo var. australis existe una familia con valor mínimo de 3.61 dentro de la población El Piñón, Hgo. y otra dentro de la población de Patoltecoya, Pue. con valor máximo de 5.85 , lo que origina una diferencia de 2.24 unidades. Por su parte en el grupo de la var. greggii el valor mínimo a nivel familias corresponde a Las Placetas, N.L. con 3.02 y el máximo a Puerto los Conejos, Coah.N.L. con 4.65, lo que da una diferencia de 1.48 unidades de color del follaje entre familias, en el mismo grupo la población que mostró mayor diferencia entre familias fue el de Las Placetas, N.L., cuyo valor mínimo y máximo correspondió a 3.02 y 4.12, respectivamente. Estas diferencias indican que los árboles de $P$. greggii se comportan de forma variable al haber evolucionado en distintos niveles de acidez en el suelo.
A los 16 meses de edad, una vez disminuido el $\mathrm{pH}$, la tonalidad amarillenta del follaje disminuyó. Sin embargo, siempre las poblaciones de la var. greggii, norte de la distribución, tuvieron tonalidades menos amarillentas (Tabla 3). Donahue (1990) encontró diferencias de desarrollo entre poblaciones de $P$. greggii, al parecer en relación con la baja elevación y presencia de suelos ácidos de las poblaciones de Querétaro e Hidalgo (var. australis) y con la alta elevación y suelos ligeramente alcalinos de las poblaciones de Coahuila y Nuevo León (var. greggii).

En lo que respecta a las familias, a los 16 meses de edad, se encontró que en el grupo sur, la variabilidad entre familias fue de 1.70 unidades, esto es la diferencia entre la familia con el tono más oscuro, una de Xochicoatlán, Hgo., con el tono más amarillento, una familia de Minas San Francisco, Hgo. fue de 1.70 unidades. En el grupo norte, Puerto San Juan, Coah. tuvo la familia con el valor mínimo y Las

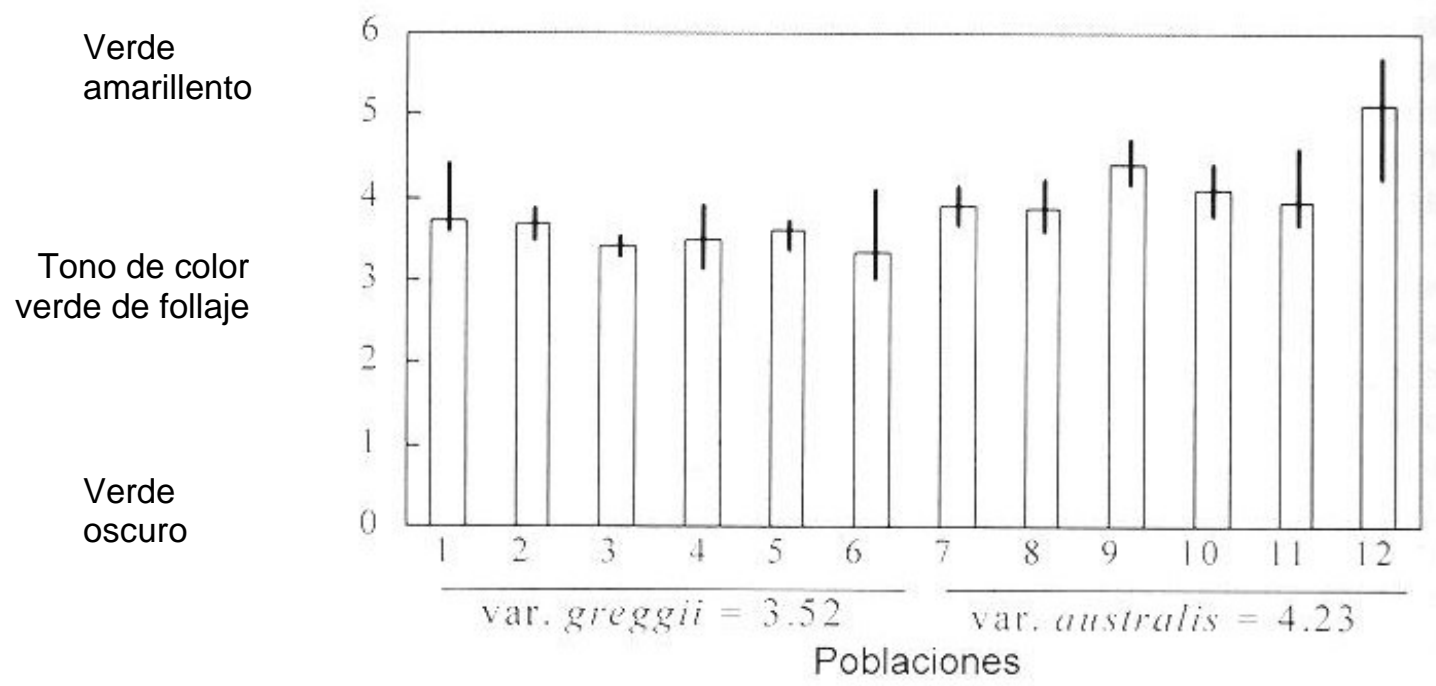

Figura 2. Valores promedio por procedencia (barra) y promedios de las familias extremas para la variable color del follaje en plántulas de Pinus greggii. El análisis de varianza indicó diferencias significativas con $p=0.01$ entre poblaciones y familias. Poblaciones: 1) Puerto Los Conejos; 2) Santa Anita; 3) Puerto San Juan; 4) Los Lirios; 5) Jamé; 6) El Madroño; 7) El Piñón; 9) Molango; 10) Xochicoatlán; 11) Minas San Francisco y 12) Patoltecoya. 
Placetas, N.L. la familia con el valor máximo, cuya diferencia fue de 1.61. Se observa que aunque pequeñas, existen mayores diferencias del color del follaje en la var. australis con relación a la var. greggii, misma situación que se observó en la emergencia de plántulas.

\section{Altura total}

El análisis de varianza de la altura de las plantas a ocho y 16 meses de edad (Tabla 3), indica que la variación entre y dentro de poblaciones resultó altamente significativa en ambas edades en que se realizaron las evaluaciones; además conforme aumentó la edad de las plantas las diferencias en altura entre poblaciones se fueron incrementando.

En otras especies se han encontrado diferencias significativas para la característica altura de planta que se mide desde el primer año de edad hasta los tres años, diferencias que han sido consideradas a factores genéticos (Wright y Bull, 1962; Squillace, 1966; Read 1980). Así, Ruiz (1989) encontró diferencias significativas entre y dentro de las poblaciones de Pinus greggii de El Madroño, Qro. y Laguna Atezca (Molango), Hgo. Por otra parte Dvorak y Donahue (1993) señalan que en un ensayo establecido en Brasil, plántulas de un año de edad de $P$. greggii del centro de México eran $150 \%$ más altas que plántulas del norte de México. Ellos señalaron que probablemente la menor velocidad de crecimiento de las poblaciones del norte sea una adaptación a las condiciones adversas en que habitan (Tabla 1).

El análisis de la altura total también manifiesta la separación de los grupos norte-sur a la edad de ocho y 16 meses (Tabla 3). El grupo de la var. australis mostró de manera general crecimientos mayores en las mediciones. Resultados similares encontró Capó et al. (1993) con plántulas de 14 meses de edad. Dentro de este grupo, la población de Minas San Francisco, Hgo. se diferenció del resto por tener los valores más bajos (Fig. 3, Tabla 1). Esta falta de vigor en el crecimiento puede ser atribuible a posibles problemas por endogamia (Sorensen y Miles 1978), ya que esta población es pequeña y el arbolado está muy disperso, lo que puede afectar una polinización efectiva, como antes fue mencionado. En cuanto a los valores de la altura para las familias (Fig. 3) se aprecia en las poblaciones de la var. australis las mayores diferencias entre familias dentro de poblaciones las presentó Xochicoatlán, Hgo.

La var. greggii presentó menor crecimiento y menos variabilidad en altura que la var. australis (Fig. 3). Por ejemplo, la diferencia a 16 meses de edad entre poblaciones fue de 1.6 en la var. greggii, mientras que en la var. australis fue de 6.3 $\mathrm{cm}$. Comparando a nivel de medias de familias, la diferencia entre la familia de altura mayor y la familia de altura menor es de $5.8 \mathrm{~cm}$ en la var. greggii $(16.5 \mathrm{~cm}$ en Jamé y $10.7 \mathrm{~cm}$ en Las Placetas), y de $11.2 \mathrm{~cm}$ en la var. australis $(14.2 \mathrm{~cm}$ en Minas San Francisco, $25.4 \mathrm{~cm}$ de El Madroño). Estos resultados parecen estar asociados a la variabilidad ambiental de sus poblaciones. Las poblaciones de la var. greggii habitan ambientes más semejantes entre sí que las poblaciones de la var. australis, además de que estas últimas están más dispersas entre sí (Tabla 1). Si estas diferencias se mantienen a edad adulta, se podrían obtener mayores ganancias por altura al seleccionar a nivel familias en la var. australis que en la var. greggii. Aún cuando el grupo norte y sur se encuentran bien diferenciados en sus valores medios de altura por procedencia, es posible encontrar familias de diferentes grupos con crecimientos similares. Así por ejemplo, a los 16 meses de edad, la familia con valor mínimo $(16.8 \mathrm{~cm})$ en Xochicoatlán, Hgo. tiene solamente $0.3 \mathrm{~cm}$ de diferencia 


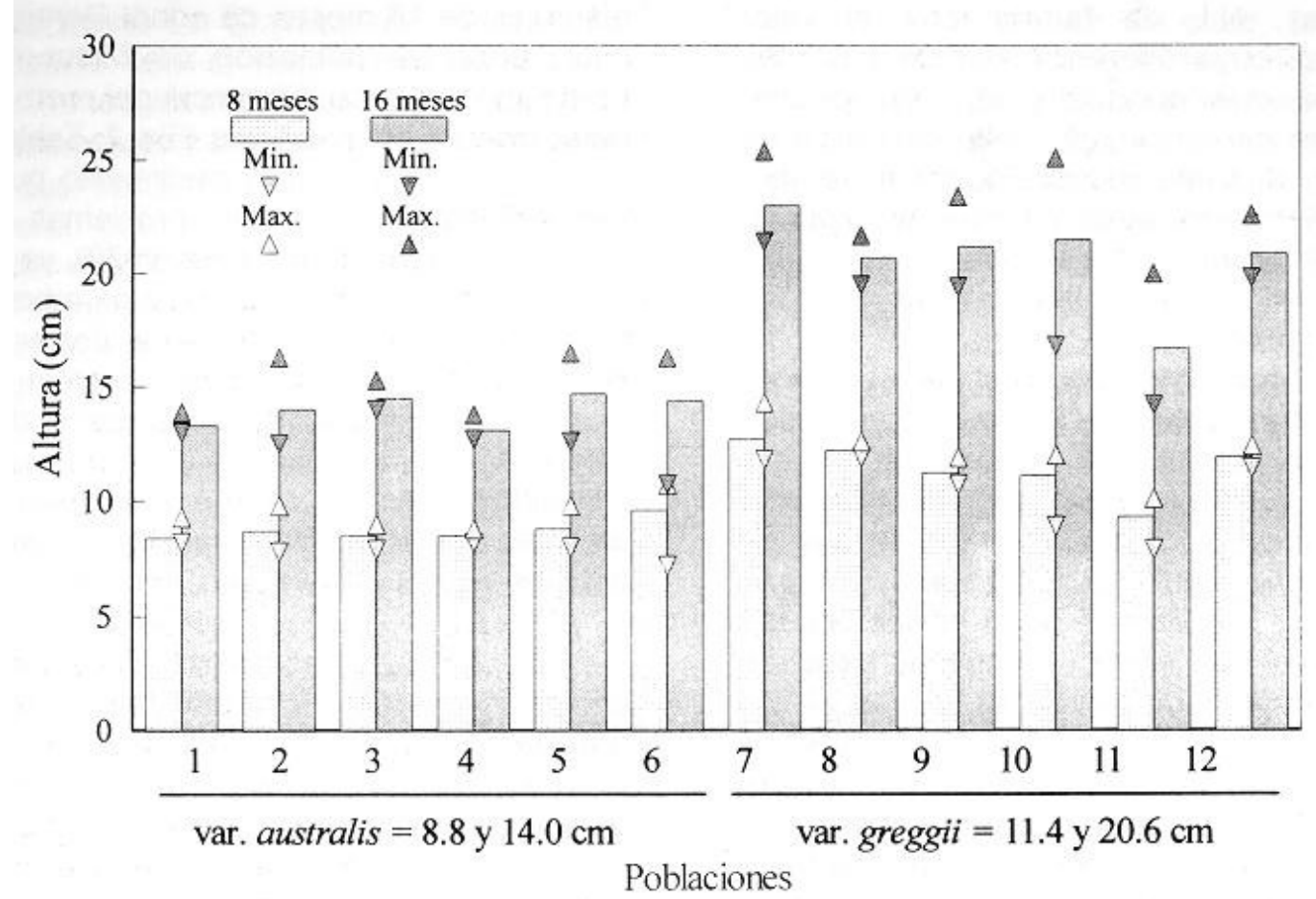

Figura 3. Valores promedio por procedencia (barra) y promedios de las familias extremas (Mín. y Máx.) para la variable altura total en plántulas de Pinus greggii sometidas a riego con agua alcalina hasta los ocho meses. Evaluación a 8 y 16 meses. El análisis de varianza indicó diferencias significativas con $p<0.01$ entre poblaciones y entre familias en las dos fechas de medición. Poblaciones: 1) Puerto Los Conejos; 2) Santa Anita; 3) Puerto San Juan; 4) Los Lirios; 5) Jamé; 6) El Madroño; 7) El Piñón; 9) Molango; 10) Xochicoatlán; 11) Minas San Francisco y 12) Patoltecoya.

con la familia de valor máximo (16.5) en Jamé, Coah., sin embargo, en sus valores medios ambas poblaciones presentan considerables diferencias. Estas similitudes entre familias pueden ser de utilidad para seleccionar árboles con una velocidad de crecimiento semejante pero adaptados a un ambiente distinto, por ejemplo el nivel de acidez del suelo.

\section{Componentes de varianza}

Utilizando los valores de la suma de cuadrados tipo III, se calcularon los componentes de varianza (Tabla 4). En la mayoría de las características se aprecia una mayor variabilidad entre las poblaciones que entre las familias dentro de las poblaciones, hecho que puede deberse a las claras diferencias entre las variedades de $P$. greggii. Los valores del error experimental resultaron ser los más altos para el color de follaje y la altura a ocho meses, parte de ello se debe a la variación entre plantas de una misma familia.

En cuanto al número de días para la emergencia de las plántulas se encontró que la mayor variación se presentó entre poblaciones con $59 \%$, después $23 \%$ corresponde al error experimental y la variación entre familias obtuvo $18 \%$ del total de la variación. En el color del follaje se pudo observar una gran variación entre plantas dentro de la misma familia, aunque la variación debida a poblaciones se incrementó ligeramente con la edad de 12.9 a $17.0 \%$. King (1965), encontró en plantas de cuatro años de edad, que el color del follaje estuvo asociado con el origen de las poblaciones de Pinus 
sylvestris L., señalando que hasta un 50\% del componente de variación provino de la procedencia de la semilla.

En lo que respecta a la altura, el porcentaje de variación a nivel poblaciones aumentó considerablemente, de $35.5 \%$ a ocho meses a $58 \%$ a los 16 meses de edad, mientras que la variación entre familias dentro de poblaciones se mantuvo estable con $8 \%$ en las dos fechas de medición. Esto indica que conforme crecieron las plantas, las diferencias entre poblaciones (y variedades) se hicieron mayores. Este incremento en el componente de varianza de la variable altura entre poblaciones se ha manifestado en algunos ensayos como el de Ruiz (1989) que encontró diferencias altamente significativas entre poblaciones y entre familias dentro de estas para plántulas de seis meses de edad de $P$. greggii. Posteriormente con las mismas plantas,
Castellanos y Ruiz (1993) señalan que a cuatro años de edad no se encontraron diferencias para las familias dentro de poblaciones, pero sí entre las poblaciones.

\section{Heredabilidad}

De manera general se observa que existe un mayor control genético en la altura total de plantas que en el color del follaje (Tabla 5). El color del follaje tuvo valores relativamente bajos de heredabilidad a nivel individual, sin embargo, para familias resultó relativamente alta con valores más cercanos a los observados para la característica altura de la planta. En la variable altura total se observa un aumento en los valores de heredabilidad a nivel individual conforme aumentó la edad de las plantas, lo cual es ratificado por Nienstaedt y Riemenschneider (1985); aunque los valores más altos siempre correspondieron a las familias.

Tabla 4. Componentes de varianza en porcentaje para cinco características en plántulas de Pinus greggii Engelm.

\begin{tabular}{||l|c|c|c|c||}
\hline \multicolumn{1}{|c|}{ VARIABLE } & PROCEDENCIA & FAMILIAS & ERROR & TOTAL \\
\hline Días a emergencia $^{1}$ & 59.0 & 17.8 & 23.2 & 100 \\
\hline Color a ocho meses & 12.9 & 2.8 & 84.3 & 100 \\
\hline Color a 16 meses & 17.0 & 2.8 & 80.2 & 100 \\
\hline Altura a ocho meses & 35.5 & 7.7 & 56.8 & 100 \\
\hline Altura a 16 meses & 58.0 & 8.0 & 34.0 & 100 \\
\hline
\end{tabular}

1. Número de días para alcanzar el $50 \%$ de la emergencia de plántulas.

Tabla 5. Valor de la media armónica (n) del número de plantas por parcela, varianzas estimadas, y valores de heredabilidad individual $\left(h^{2}\right)$ y de las medias de familia $\left(\mathrm{h}_{\mathrm{f}}^{2}\right)$, para color de follaje y altura de plántulas de Pinus greggii a dos edades.

\begin{tabular}{|l|c|c|c|c|c|c||}
\hline \multicolumn{1}{|c|}{ VARIABLE } & $\mathrm{N}$ & $\sigma^{2}{ }_{E}$ & $\sigma^{2}{ }_{B F(P)}$ & $\sigma_{F(P)}^{2}$ & $H^{2}$ & $H^{2}{ }_{F}$ \\
\hline Color a 8 meses & 13.33 & 1.1601 & 0.0677 & 0.0388 & 0.12 & 0.56 \\
\hline Color a 16 meses & 9.65 & 0.9258 & 0.0827 & 0.0319 & 0.12 & 0.47 \\
\hline Altura a 8 meses & 13.67 & 455.0300 & 46.6700 & 61.6400 & 0.44 & 0.79 \\
\hline Altura a 16 meses & 9.65 & 1001.9500 & 220.2600 & 236.3200 & 0.65 & 0.78 \\
\hline
\end{tabular}


Aunque las estimaciones de heredabilidad se realizan con cierto error, las proporciones obtenidas son una indicación relativa del control genético existente (Zobel y Talbert, 1984). Por lo tanto, aún cuando los valores de heredabilidad de familias para color del follaje son moderadas, podrían ser de utilidad para seleccionar familias dentro de poblaciones con mayor tolerancia a valores de $\mathrm{pH}$ cercanos al neutro, los cuales predominan en suelos desprovistos de cubierta forestal. Además se podrían seleccionar familias del norte (var. greggii) con crecimientos similares o mayores a las familias del sur (var. australis) que se desarrollan en $\mathrm{pH}$ ácidos. Lo anterior indica que obtener ganancias genéticas parece ser posible por medio de la selección a nivel de individuos de las mejores familias.

\section{DISCUSIÓN GENERAL}

Es evidente, debido a las diferencias encontradas en el análisis, que existe una variación en Pinus greggii a lo largo de su distribución natural y que fortalece la propuesta de la existencia de dos grandes grupos de poblaciones que corresponden a las variedades greggii y australis según Donahue y López (1999), y que forman parte de la variación de la especie. Estas diferencias en grupos 0 variedades taxonómicas pueden estar asociadas a procesos evolutivos asociados a la adaptación a ambientes diferentes, por ejemplo el clima, ya que en el extremo sur de distribución natural de la especie el clima varía de templado-subhúmedo a subtropical-húmedo, que corresponden a los estados de Puebla, Hidalgo y Querétaro, mientras que en la parte norte de manera general el clima es semiáridofrío, en los estados de Coahuila y Nuevo León. Otro factor importante, es el aislamiento que cada población presenta, situación que pudo haber llevado, y estar sucediendo aún, a una diferenciación fenotípica y genotípica bastante clara, como para identificarse entidades taxonómicas diferentes.
Las poblaciones de la var. greggii tuvieron en las dos fechas tonalidades del follaje verde más obscuro que las de la var. australis. Es probable que esta característica sea ya una adaptación a las severas condiciones ambientales del norte del país (Tabla 1). Por otro lado, fue posible observar que el cambio en el $\mathrm{pH}$ del substrato utilizado no afectó en forma drástica y permanente en el crecimiento de las plantas en vivero, sino que posiblemente actuó modificando la disponibilidad de algunos elementos nutricionales. Así, cuando la acidez del sustrato disminuyó, es decir cuando el pH tendió a la neutralidad, las plantas debieron haber manifestado síntomas de deficiencia de algún elemento aún cuando éste se encontrara presente, debido al problema de la baja disponibilidad de los elementos para ser absorbidos por las plantas. Aunque no fue posible probar dos niveles de $\mathrm{pH}$ al mismo tiempo, el hecho de que el color de follaje más oscuro sea asociado a un estado sano y de crecimiento vigoroso, se puede concluir que $P$. greggii se desarrolla mejor en condiciones ácidas que en medios tendientes a neutros, tal como lo mencionan Ruiz (1989), Donahue (1990) y García (1990).

Los valores de heredabilidad fueron relativamente altos, aumentando estos valores conforme aumenta la edad de las plantas. De esta forma, es claro que existe cierto control genético que puede acelerar la selección de poblaciones, familias e incluso individuos dentro de familias, desde edades muy tempranas, siendo necesario corroborar los resultados en campo y a mayor edad.

\section{CONCLUSIONES}

Se encontraron diferencias significativas entre poblaciones y entre familias dentro de poblaciones en las variables sobrevivencia, días a emergencia, color del follaje y altura en las plántulas de doce poblaciones de $P$. greggii, aunque la variación entre poblaciones 
fue ligeramente mayor que entre las familias dentro de las poblaciones.

Con las características analizadas $P$. greggii manifestó la separación de dos grupos, uno con características de poblaciones relativamente uniformes en el norte y otro con ligeras variaciones dentro del grupo en el sur de su distribución, mismas que concuerdan con la separación de la taxa en dos variedades.

La disminución de la acidez en el sustrato tuvo un efecto negativo en las plantas, manifestándose una clorosis en el follaje, sin embargo, las poblaciones del sur presentaron tonalidades más amarillentas en ambas condiciones de $\mathrm{pH}$ del sustrato a lo largo del estudio. Aunque de manera general las plantas de $P$. greggii producidas en vivero se desarrollaron mejor en $\mathrm{pH}$ ácidos.

En las variables color del follaje y altura a diferentes edades se encontró un control genético moderado, tanto a nivel individual como de las medias de familia. El control genético para altura aumentó su valor con la edad de las plantas.

\section{RECONOCIMIENTOS}

Este trabajo se llevó a cabo parcialmente bajo el financiamiento del proyecto CONACyT P220-CCOR-904153 y sirvió como requisito de la licenciatura del segundo autor.

\section{REFERENCIAS}

Capó A., M.A., R. López A. y E. Cornejo O. 1993. Crecimiento de Pinus greggii en suelo de ocho localidades. In: I Congreso Mexicano sobre Recursos Forestales. Saltillo, Coah. p. 75.

Castellanos B., J.F. y M. Ruiz M. 1993. Introducción de Pinus greggii Engelm., en la Mixteca Alta
Oaxaqueña. Folleto de investigación No. 1. INIF, SARH. Centro de Investigación Pacífico Sur. Yanhuitlán, Oaxaca, Méx. 18 p.

Donahue, J.K. 1990. Geographic variation in Pinus greggii Engelm., in relation to soil acidity. M.S. Thesis. North Carolina State Univ. Raleigh, EUA. 70 p.

Donahue, J.K. y J. López U. 1999. A new variety of Pinus greggii (Pinaceae) in Mexico. Sida 18(4):1103-1113.

Dvorak, W.S. y J.K. Donahue. 1993. Reseña de Investigaciones de la Cooperativa CAMCORE 1980-1992. CAMCORE. North Carolina State Univ. Raleigh. EUA. p:26-28.

Falconer, D.S. y T.F.C. Mackay. 1996. Introduction to Quantitative Genetics. 4th ed. Longman, Exxex. Gran Bretaña.. $464 \mathrm{p}$.

García L., E. 1990. Efecto del sustrato y fertilización en el crecimiento de Pinus greggii Engelm. Tesis profesional. Di.Ci.Fo. U.A.Ch. Chapingo, Méx. 76 p.

INEGI. 1980. Atlas nacional del medio físico. Carta de climas. Instituto Nacional de Estadística, Geografía e Informática, México D.F.

King, J.P. 1965. Seed source $x$ environment interactions in Scotch pine. II. Needle lenght and color. For. Abstr. 26 (4):550.

Landis, T.D., R.W. Tinus, S.E. McDonald y J.P. Barnett. 1989. Seedling nutrition and irrigation. Vol. 4. The container tree nursery manual. USDA, Agricultural Handbook 674. 111 p.

López U., J., J. Jasso M., J.J. Vargas H. y J.C. Ayala S. 1993. Variación de características morfológicas en conos y semillas de Pinus greggii 
Engelm. Agrociencia serie Recursos Naturales Renovables 3(1): 81-95.

Lutz, H.J. y R.F. Chandler. 1949. Forest soil. John Wiley and Sons. Nueva York. p: 361-371.

Malagón L., M. 1990. Estudio de variación morfológica y de la germinación en cuatro procedencias de Pinus greggii Engelm. Tesis profesional. ENEP. UNAM. México. 105 p.

Matheson, A.C., J.C. Bell y R.D. Barnes. 1989. Breeding systems and genetic structure in some Central American pine populations. Silvae Genetica 38(3-4):107-113.

Nienstaedt, H. y D.E. Riemenschneider 1985. Changes in hereditability estimates with age and site in white spruce, Picea glauca (Moench) Voss. Silvae Genetica 34(1): 34-40.

Pritchett, W.L. 1986. Suelos Forestales: Propiedades, conservación y mejoramiento. Limusa, México p: 129-149.

Read, R.A. 1980. Genetic variation in seedling progeny of ponderosa pine provenances. Forest Science. Monograph No. 23. 59 p.
Ruiz M., M. 1989. Variación de caracteres juveniles de 42 familias de procedencias de Pinus greggii en 3 tipos de sustrato. Tesis Profesional. Di.Ci.Fo. U.A.Ch. Chapingo, México. 91 p.

Ruiz G., C. y E. Velasco B. 1994. Crecimiento y distribución de biomasa en plantas de Pinus engelmanii Carr., bajo dos niveles de humedad del suelo. Tesis Profesional. Di.Ci.Fo., U.A.Ch. Chapingo, Méx. 109 p.

SAS. 1988. SAS/STAT guide for personal computers. SAS Institute Inc. Cary, N.C. EUA. 378 p.

Séguy, E. 1936. Code Universel Des Couleurs P. Lechevalier. Paris. 68 p.

Sorensen, F.C. y R.S. Miles. 1978. Cone and seed weight relationship in Douglas-fir from western and central Oregon. Ecology 59 (4): 641-644.

Squillace, A.E. 1966. Geographic variation in slash pine. Forest Science Monograph No. 10. 56 p.

Wright, J.W. y W.I. Bull. 1962. Geographic variation in European black pine twoyear-results. Forest Sci. 8(1):32-42.

Zobel, J. B. y J.T. Talbert. 1984. Applied forest trees improvement. John Wiley and Sons, Nueva York. 505 p. 